\title{
Computerized environment for People with Serious Disability
}

\author{
Chao Zhang, Haruo Nakashima, Motohiro Tanaka, Shunji Moromugi, Takakazu Ishimatsu \\ Graduate school of Science and Technology \\ Nagasaki University \\ Nagasaki Japan \\ bb52311103@cc.nagasaki-u.ac.jp
}

\begin{abstract}
Persons suffering from intractable disease like ALS (Amyotrophic Lateral Sclerosis) and spinal cord injured present symptoms of muscular deteriorations. These patients are often obliged to use artificial respirators once the breathing action becomes impossible. It is important to understand that using the artificial respirator means that uttering voice becomes impossible. For human being talking and discussing with friends using voice is necessary to enjoy his life with family and friends. In order to support their communication, computerized environment is proposed, where various home electric appliances can be controlled by computer. The computer has original vision input device. A distinct advantage of this computerized environment is that the all devices can be settled apart from the bed of the patient.
\end{abstract}

Keywords-component Image-processing; Infrared; Disability; Independent-life

\section{INTRODUCTION}

Everybody should be able to enjoy his independent life. However, patients suffering from ALS (Amyotrophic Lateral Sclerosis) or cerebral infarction are difficult to live independent lives. They need intensive cares by care-workers. As a typical symptom of ALS patients their muscular functions deteriorate rapidly. In two or three years after the onset of the disease, patients are obliged to use artificial respirators. Once they start to use the artificial respirator, uttering voice becomes impossible. In such a physical situation, they are usually confined to bed and physical ability are limited to slight movements of head, fingers, lips or eyes. Therefore, their relationships with society are extremely limited. However, recent developments of computer technology and network technology brought great benefits to these peoples with physical disability.

In this study we focused on the development of computerized environment for the patient as shown in Fig.1.

The patient shown in Fig. 1 is spine injuries suffered by a falling accident in 2011. His limbs are paralyzed. Also he cannot utter voices because of artificial respirators. All his remaining physical activities are limited only above his neck. In order to enable him to operate computer, he uses an original head mount computer input device as shown in Fig.1. In side the head mount computer input device an acceleration sensor and a pressure sensor are mounted. The acceleration sensor is used to detect the orientation of his head and the pressure sensor to detect the chewing action by detecting the swelling up at the temple. Using this head mount interface he can control the cursor movements on the computer display and also activate click action of the computer mouse.

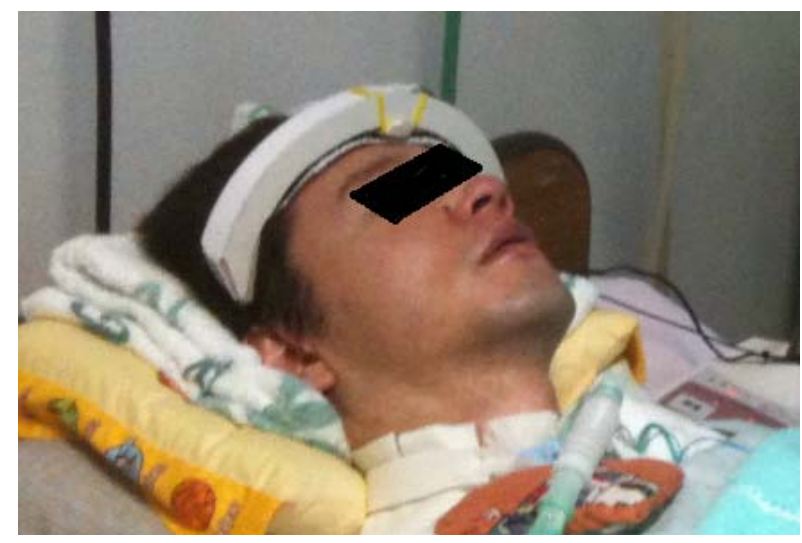

Fig.1 Patient of spinal cord injured

His head mount interface is effective for his life. However, wearing such a interface is not comfortable. Another computer input device was expected for him. Computer input devices are categorized as a stand type and a wearable type [1]. The stand type devices include touch switches, laser sensors and vision sensors. These devices are usually attached to the bed or table and used to detect some body movements. The wearable type devices introduce sensors to detect acceleration, angle, strain and EMG (Electromyogram) those are attached to the patient's body. These sensors measure physical movement or condition of the patient body. And input signal is generated based on these data measured.

Input devices to use a vision sensor have distinguished advantages over the other devices that the vision devices can be applied to person with serious physical disability [2], [3]. The vision device can be settled apart from the bed. This means care workers can be free from annoying setting task, wires and mechanical parts around the bed. Another advantage is that the vision device can be applied to detect various body movements like lips, fingers, mouth, eyebrow as well as eyeball. It is also important to note that the vision devices can be applicable to various physical ability of the user. In the first stage of the 
intractable disease like ALS the patient can move his head. But after the physical situation becomes serious, he sometimes can move only his eyeball or eyebrow. Of course, the situation depends on the patient. A feature of the vision device is that the vision device is readily applicable to various physical situations by renewing the image processing software.

Another problem for people with physical disability to live independent life, various supporting machines and devices are required. Electric appliances like TV and air conditioning system are ready to control using infrared controller. But an emergency call, bed controller and also telephone are not ready to control. In this paper we propose a device to enable controlling many kinds of devices and machines.

In this paper a vision input device is proposed. This device uses slight head movements as a positioning signal of the computer cursor. And the movements of the lips and eyebrows are used as switching signal. A feature of the input device is that function of the device can be adaptive to the upgrading and also deterioration of the user's symptom. The vision input device corresponds to a two-dimensional pointing device [5]. This input device requires the operator's remaining ability to move his or her head two-dimensionally.

This device is incorporated into the computer system to support communication of the patient. In order to test the applicability, these supporting devices are applied to a spinal cord injured. He injured his spinal cord in an accident in 2008. Due to the accident, all his possible physical movements were limited only above his neck. In addition, he couldn't utter voices and breathe because of the damage on the nerves related to breathing actions. Our devices are developed to support his independent life.

\section{CONFIGURATION OF COMPUTERIZED ENVIRONMENT}

We propose a computerized environment as shown in Figure 1. The system is composed of a vision input device, an infrared signal emitter and infrared controllers. The infrared signal emitter can control electric appliances like TV, airconditioning system and light. The infrared signal emitter also can control other devices and machines via the infrared controllers. The infrared controllers can control the devices and machines based on the infrared signal detected.

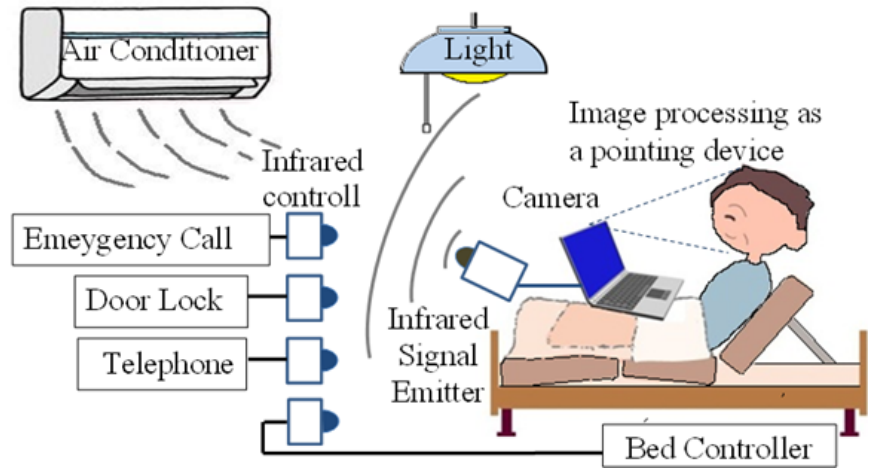

Fig. 2 Configuration of life support system,
A feature of the support system is that all the machines and electric appliances are connected with the computer via infrared signals. Due to this feature, the computer is free from bothersome cables.

The vision camera installed in the computer is used to detect the head movement of the user. By processing the sequential image of the head, intentional movements of the head, eyes, mouth and eyebrows are extracted. Once the intentional movements are detected, the personal computer is controlled based on these movements data. Physical movements of head or lips are correlated with the cursor movements on the display monitor considering the physical conditions of the user. In case of an ALS patient in Nagasaki a vision device is used to detect the position of his head where muscular movement is active. According to the head movement, the computer cursor on the computer display is moved. And in case he wants to activate the icon on the display monitor, all he has to do is to move the computer cursor on to the icon and to open and close his mouse slightly. Immediately after that, the icon on the display monitor is activated. In order to enable efficient communication with other people, a specialized communication software is installed whose main functions are to write texts and to control various home electric appliances. Using these functions, the user can communicate with family members at home, talk with them and enjoy TV programs he wants.

\section{Pointing DeVice to Use Vision}

Currently conventional computers can be operated only by the computer mouse operations, like two-dimensional pointing and click operations. In case the user with upper limb disability is able to move his head intentionally, the head movement can be used to control the computer cursor.

The head movement can be detected by the following two methods.

\section{Method 1) Using two marks on the face}

In this method the user is requested to wear at least two marks on his face as shown in Fig.3. Marks should have specialized color pattern to re detected easily from the background image. In this case, the mark is a green and blue circle like Fig 3: One is put on the nose, and the other is put under the lips. For this preparation, we use OpenCV program to detect the marks, and confirm the position of marks with a home computer. When the patient changes his face direction, the cursor will move in the according to the movement of the marks. Furthermore, the patient can open and close his mouth to achieve a cursor click.

A distinctive advantage of the method is that the method is robust to lighting conditions. This feature is important to the image processing technique. 


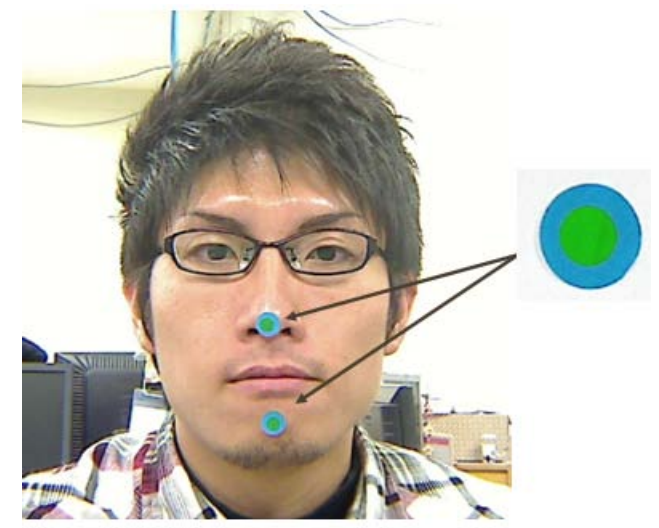

Fig 3. the mark on the face

Color image composed of RGB are converted to YCC. Pixels to have human skin color can be plotted onto C1-C2 scales as shown in Figure 2, where $\mathrm{C} 1$ and c2 scales are defined as follows.

$$
\begin{aligned}
& \mathrm{C} 1=0.701 \mathrm{R}-0.587 \mathrm{G}+0.886 \mathrm{~B} \\
& \mathrm{C} 2=-0.299 \mathrm{R}-0.587 \mathrm{G}+0.886 \mathrm{~B}
\end{aligned}
$$

Considering the enclosing boundary in Figure 2, we adopted the region of the skin area as follows.

$$
\begin{aligned}
& \mathrm{C} 1>0 . \\
& \mathrm{C} 1<-\mathrm{C} 2+40 \\
& \mathrm{C} 1>-0.6248 \mathrm{C} 2 \text { (where } \mathrm{H} \\
& 15<\mathrm{S}<50 \\
& \text { where } \\
& \mathrm{H}=\arctan (\mathrm{C} 1 / \mathrm{C} 2) \\
& \mathrm{S}=\operatorname{sqrt}\left(\mathrm{C}^{2}+\mathrm{C}^{2}\right)
\end{aligned}
$$$$
\text { C1 }>-0.6248 C 2 \text { (where } \mathrm{H}<148 \text { degree) }
$$

In Figure 4 an example of skin detection is shown. In the right picture in the figure only the pixels to have skin color are denoted as white pixel.

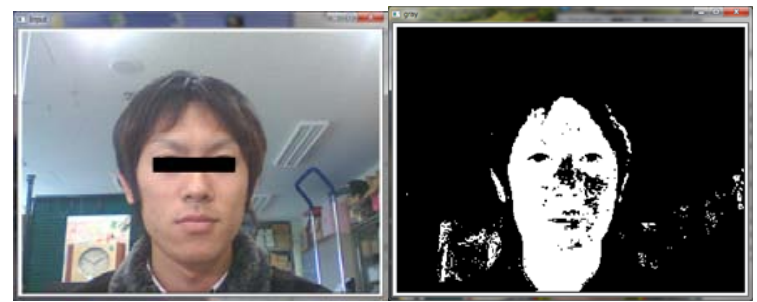

Fig 4. Extraction of skin.

\section{Method 2) Detection without marks}

In this method, we use camera to catch face of the patient and send this image to OpenCV program.

Once the skin pixels in the image are detected, the face area can be estimated. After the estimation the face area are converted to binary image as shown in Figure 5. The binary image of the face can be obtained using luminance equation as follows.

$$
\mathrm{Y}=0.299 \mathrm{R}+0.587 \mathrm{G}+0.114 \mathrm{~B}
$$

Based on this scale, the face image can be binaries as shown Figure 5. On to this binary image we conducted the labeling software program. Comparing the size of the white area, and also considering eye and eyebrow, mouth can be detected where small clusters of the white pixel are neglected.

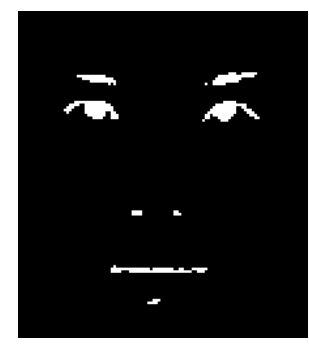

Fig 5. Binary image of the face

Considering the variations of the raster coordinates of the eye, eyebrow and mouth, various operation of the computer can be conducted.

\section{EVALUATION OF VISION DEVICE}

A vision sensor (Camera with $480 \mathrm{H} \times 640 \mathrm{~V}$ resolution) was settled $50 \mathrm{~cm}$ apart from the patient to detect his head movement. And the functional ability was evaluated as follows.

\section{A. Evaluation of Pointing Accuracy}

Pointing accuracy of the vision input device was evaluated by healthy examinees without any physical disability. The pointing device was used to locate the mouse pointer on the computer display by using head movements. Examinees were requested to move his head so that the mouse cursor traces the rectangular shape in the computer display. And also the examinees were requested to conduct click action at all four corners. The click action means opening his mouth. The width and height of the target rectangle on the computer display was the length of 500 pixels as shown in Figure 6. In these experiments our vision device were tested twice. And a conventional mouse device and touchpad device were tested .Required times to trace the rectangle and also click at every four corners were shown in TABLE I. The required time of the proposed device is less than three times of conventional devices.

Also average tracking error during the tracing tasks is shown in TABLE II. The average pointing accuracy of the proposed device was around 4 to 13 pixels. 


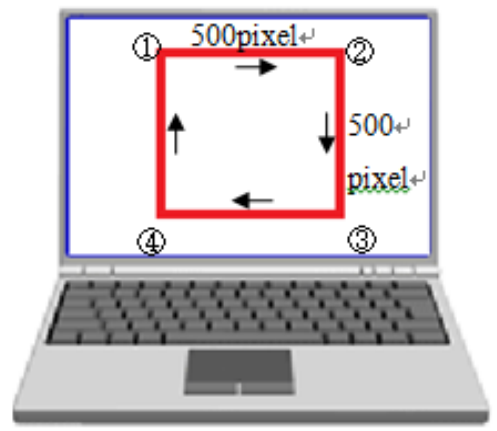

Fig 6. Tracing test along rectangle

TABLE I. Time to trace the rectangle.

\begin{tabular}{|l|c|c|c|c|}
\hline Examinee & A & B & C & D \\
\hline Proposed device(1 ${ }^{\text {st }}$ test $)$ & 64 & 88 & 53 & 53 \\
\hline Proposed device(2ne test) & 63 & 71 & 63 & 91 \\
\hline Touchpad device & 23 & 34 & 46 & 36 \\
\hline Mouse device & 20 & 21 & 29 & 57 \\
\hline
\end{tabular}

TABLE II. Average tracking error to trace the rectangle.

\begin{tabular}{|l|c|c|c|c|}
\hline Examinee & A & B & C & D \\
\hline Proposed device( $\left(^{\text {st }}\right.$ test $)$ & 13.11 & 2.73 & 3.88 & 4.21 \\
\hline Proposed device(2nd test) & 10.12 & 5.94 & 8.15 & 6.50 \\
\hline Touchpad device & 1.64 & 1.56 & 1.64 & 2.31 \\
\hline Mouse device & 5.45 & 2.36 & 2.45 & 2.46 \\
\hline
\end{tabular}

\section{B. Evaluation of Character input.}

The vision input device was applied to communication software named Hearty Ladder. A computer display of the communication software is shown in Figure 7. In the lower half of this display many buttons were arranged. To each button one character or one function was allocated..

By selecting the desired characters sequentially, desired text could be completed as shown in the upper half of the display where the text was GOOD MORNING..

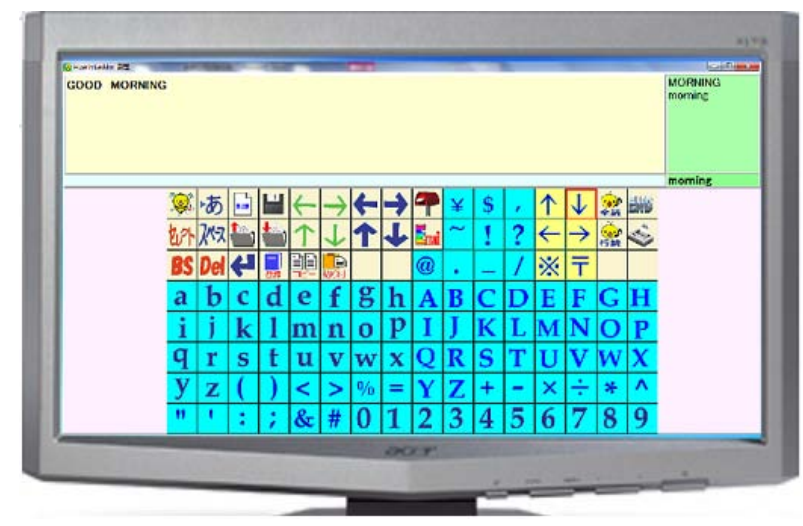

Fig.7 Computer display of communication system

Using this communication system, four examinees were requested to write the text "GOOD MORNING". TABLE III shows time required to write the text. Of course by the conventional mouse operation the text can be written in around 15 seconds.

TABLE III. Time to input text

\begin{tabular}{|l|c|c|c|c|}
\hline Examinee & A & B & C & D \\
\hline Time to input text & 134 & 156 & 165 & 142 \\
\hline
\end{tabular}

\section{APPLICATION OF SUPPORT SYSTEM}

A support system was applied to a patient with spinal cord injured. He succeeded to operate the communication system effectively using proposed input device as shown in Figure 8.and 9.

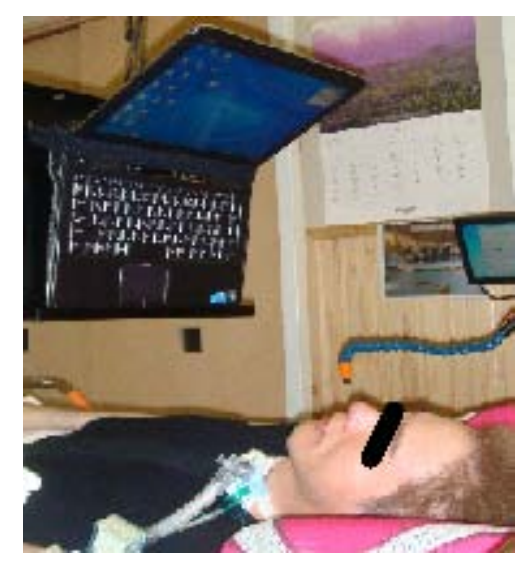

Fig.8 User with vision input device

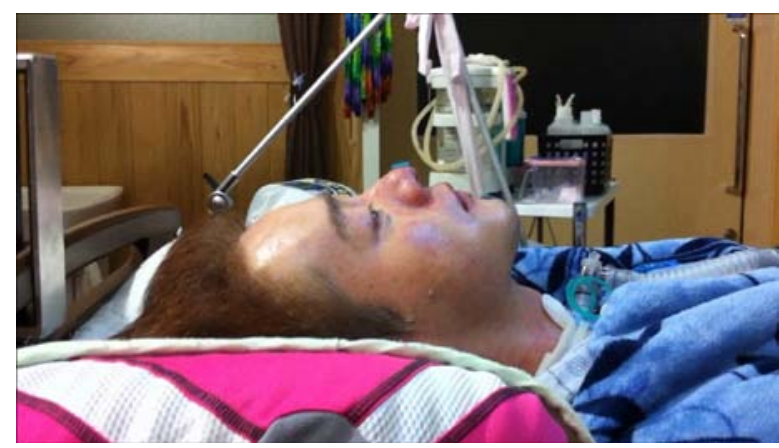

Fig.9 User with vision input device

In Figure 10. an infrared emitter is shown. And in Fig.11 a telephone which is controlled by infrared controller is shown. A feature is that the telephone can be controlled by the following steps.

Step1) Push hands-free button

Step2) Push address 1 button

These two buttons are pushed by two solenoids, which are controlled by infrared controller. After pushing these two buttons, the operator can speak via telephone. Even if the user cannot utter voice, the computer voice enables the user to 
speak via telephone. In case there is a telephone call from outside, only pushing the hand-free button enables the user to communicate with others on the telephone.

Of course pushing these two buttons sequentially can be simplified to clicking one button on the communication display.

The user could operate communication device successfully without any difficulty..

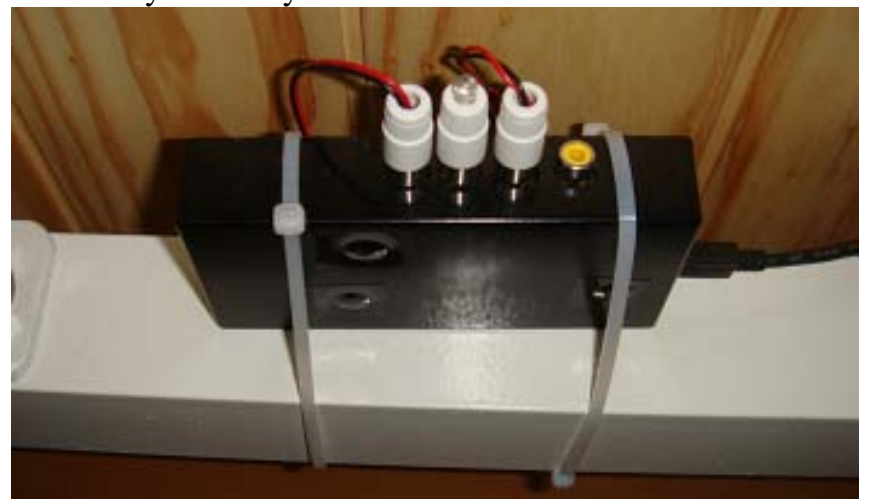

Fig 10. Infrared emitter.

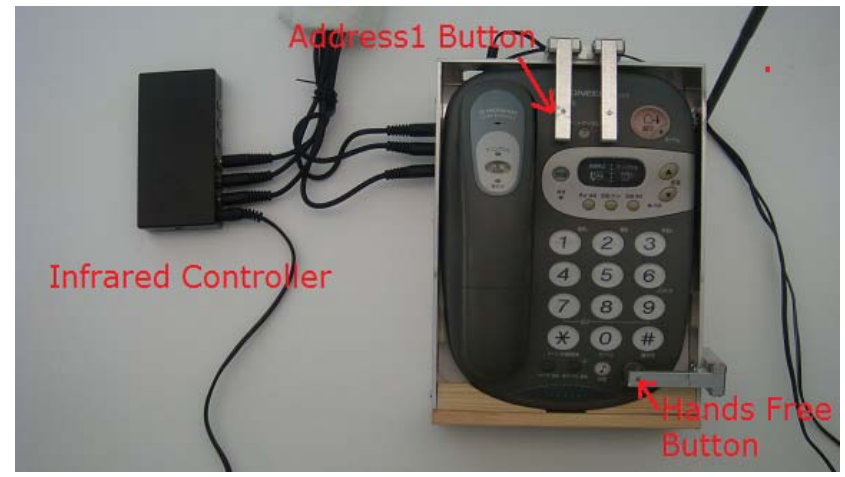

Fig 11. Telephone controlled with infrared signal

\section{CONCLUSION}

A vision input device is proposed as an effective input device which is robust to the physical abilities. A distinct feature of the vision device is that vision sensor is used as a two-dimensional pointing device. This device enabled comfortable operations of the communication system. This supporting system was applied to spinal cord injury. The patients could communicate successfully with their families and operate electric devices by using the vision input device.

\section{REFERENCE}

[1] Y.Fukuda, M.Tanaka, S. Moromugi and T. Ishimatsu, “A Communication device for disabled slight movement capabilities”, International Journal of HWRS, vol.4, No.4, December, pp.7-12, 2003.J. Clerk Maxwell, A Treatise on Electricity and Magnetism, 3rd ed., vol. 2. Oxford: Clarendon, 1892, pp.68-73.

[2] T. Ochiai, T. Ishimatsu, "Computer Input device for handicapped using vision sensor", in Proc.3rd Int. symposium on artificial life and robotics Vol.2, pp.634-637, 1997.
[3] O.Takami, T.Ishimatsu, and T.Shimomachi, Development of the environmental control system by using eyeball movements, Proceeding of the Asian Control Conference, pp.715-718, July 27-30, 1994.

[4] O.Takami, K.Morimoto, T.Ochiai, and T. Ishimatsu, Computer Interface to use head and eyeball movement for handicapped people, 1995 IEEE International Conference of System, Man and Cybernetics, Vancouver, Canada, October 22-25, 1995.

[5] H.Kawamura, N.Dohi, T.Oshima, "Experimental Production of Light Mouse using PSD sensor", Proc. of 5th Rehabilitation Engineering, Tokyo, Japan, 21-24 August, 1990, pp.233-236 